\title{
INVESTIGATION OF HEART RATE VARIABILITY BY STATISTICAL METHODS AND DETRENDED FLUCTUATION ANALYSIS
}

\author{
Galya Nikolova Georgieva-Tsaneva ${ }^{1}$
}

\begin{abstract}
The physiological signals that are recorded from different parts of the human body have a non-stationary nature and the tracking of their dynamics is an interesting research problem. This report examines Heart Rate Variability through the use of statistical methods of analysis that are traditionally used to study the functionality of the heart and via Detrended Fluctuation Analysis. The use of the technique of Detrended Fluctuation Analysis allows the investigation of short-term and long-term correlations in non-stationary Heart Rate Variability series. A study has been made of the changes in the functioning of the human heart, depending on the age. The study encompasses healthy individuals in three different age groups. The analysis of the obtained results shows a change in the correlated behavior of the investigated signals with an increase in age.
\end{abstract}

UDC Classification: 612, DOI: 10.12955/cbup.v7.1446

Keywords: Heart Rate Variability, Detrended Fluctuation Analysis, Statistical methods.

\section{Introduction}

Physiological signals, recorded by the human body, are difficult to investigate because of the nonstationarity of their nature. One of the vital functional systems of the human organism - the cardiovascular system - has been the subject of numerous scientific studies in recent years because of its key importance for the proper working of the human body. The intervals between adjacent heart beats in healthy individuals are not equal, they are constantly changing. This extremely physiological phenomenon was discovered by Haller (1757) and it marked as the beginning of interest and studying the phenomenon of Heart Rate Variability (HRV). The first results of HRV studies were on fetal records and were published in 1963 by Hon and Lee (1963), as a result of observations on the change in the variation between heart rate after stress.

Tracking of HRV dynamics through various parameters allows for predicting a high probability of death in the event of a severe reduction in parameters of HRV (associated with vegetative disorders of heart work). The phenomenon of sudden cardiac death can be predicted through Heart Rate Variability. Through HRV, various diseases such as cardiovascular disease, blood pressure, diabetes mellitus, and others can be investigated.

In the first HRV studies in the scientific literature, the difference between the minimum and maximum cardiac RR intervals, standard RR interval deviation and the analysis of short-term 5-15 minute records were calculated. With the invention of the Holter monitor, long-term observation of the activity of the cardiovascular system are provided.

HRV determines the time difference of heart rate intervals and evaluates the causes and interrelationships between sympathetic, parasympathetic and humoral regulation of the cardiovascular and nervous system. It is possible to assess the various types of stress (mental, physical, etc.) through the HRV.

Heart Rate Variability is an effective observer of the internal system for regulating the human organism. The human regulatory system is a dynamic, fast response system that defines the activities of other subsystems. HRV is a widespread non-invasive method (Georgieva-Tsaneva, 2018), that can be used in medical practice to monitoring the changes (physiological or pathological) of the regulatory system in humans. The standard established in 1996 for HRV and cardiovascular disease risk assessment provides HRV normative values for healthy individuals and brings together the results of numerous scientific studies (Malik, 1996).

HRV is used for:

- Assessment of the functional state of the human organism;

- Exploration of the vegetative balance and neurohumoral regulation of the human;

- Examination of the body's response to the stress;

- Investigation of regulation of blood circulation in due to change of external conditions;

- Creating an individual's health forecast based on the body's functional status, adaptability and regulatory mechanism of the individual systems of the human organism.

\footnotetext{
${ }^{1}$ Institute of Robotics, Bulgarian Academy of Sciences, Sofia, Bulgaria, galicaneva@abv.bg
} 


\section{Literature review}

The technology of Detrended Fluctuation Analysis (DFA) is used in the scientific literature to analyze correlations in the fluctuations in time signals. The DFA allows for the detection of long-range correlations in non-stationary data, such as ventricular fibrillation (Lin et al., 2010); autonomic nervous activity (Nakamura et al., 2005; Gronwald et al., 2019); cardiomyopathy (Mahon et al., 2002). The DFA is suitable for the analysis of physiological data as they have a non-stationary nature. In their paper Peng et al. (1995) for the first time introduced the method.

The authors Miao et al. (2006) investigate through DFA differences between normal individuals and people with pathologies in cardiovascular function, such as applied DFA to peak intervals. The time series HRV are obtained from the cardiological RR intervals (where $\mathrm{R}$ is the maximal amplitude in the electrocardiogram) and have a non-stationary nature. The authors Krishnam et al. (2005) use the DFA to study Heart Rate Variability in children with sleep disturbances during sleep. Golińska (2012) uses DFA in his article to distinguish healthy from diseased individuals.

Acharya et al. (2002) apply the DFA method for ECG signals classifying. Fractal properties in signals are explored in the article by Lee et al. (2002). Rojo-Alvarez et al. (2007) present a Lumped Parameter Model applying DFA for clinical studies of HRV: sympato-vagal and non-autonomic regulation of the nervous system. Yeh et al. (2006) investigated the management functions of the central nervous system with cardiological records obtained from healthy volunteers and with records from patients undergoing various types of neurosurgery operations; the study shows that the DFA method can distinguish the patients studied from healthy individuals. Gospodinov et al. (2016) examined HRV in healthy subjects and diabetic patients and found significant differences in the assessed parameters.

\section{Methodology}

The detrended fluctuation analysis calculates two scaling exponents for time series $\mathrm{t}(\mathrm{i})$, with length $\mathrm{N}$ $(i \in[1, N])$. The method is applied to a short-term series of HRV. The investigated cardiology series is integrated using the formula (Valencia, 2009):

$z(n)=\sum_{j=1}^{n}\left[t(i)-t_{\text {ave }}\right]$

Where:

$t(i)$ - cardiological intervals;

$n \in[1, N]$,

$t_{\text {ave }}$ - an average of the cardiological intervals $t(i)$ in all series.

The integrated data is divided into sub-series with equal length, in each of which the local trend is subtracted and the fluctuations in the resulting series are calculated by (Valencia, 2009):

$F(l e n)=\sqrt{\frac{1}{N} \cdot \sum_{n}^{N}\left[z(n)-z_{l e n}(n)\right]^{2}}$

len - length of sub-series;

$F(l e n)$ - the average fluctuations in sub-series.

Autocorrelation properties of HRV are well described by applying the DFA and determining a parameter labeled with $\alpha$. The parameter is determined by relationship on a $\log \log$ graph: $(\log (F(\operatorname{len})$ and $\log (\operatorname{len}))$. This parameter gives an evaluation of the self-similarity properties of the data under investigation, according to Golińska (2012) and Šikner (2015):

- $\alpha \in(-\infty, 0.5)$-indicates anti-correlation properties of investigated series;

- $\alpha=0.5$ - corresponds to Gaussian White Noise, the data is completely uncorrelated;

- $\alpha \in(0.5, \infty)$ - an indication of the presence of positive autocorrelation in the data (>1: correlations is not in power-law form);

- $\alpha \in(0.5,1]$ - persistent long-range power-law correlations;

- $\alpha=1$ - corresponds to the $1 / f$ distribution of the data;

- $\alpha \in[1,1.5]$ - long-range correlations;

- $\alpha=1.5$-indicates the presence of a random walk (Brownian noise).

Correlation properties of HRV in small blocks (length <16) are tested through the parameter $\alpha_{1}$ (alfa1), and for larger blocks (length >16) with parameter $\alpha_{2}$ (alfa2)(according to Leite, 2010). 
Prior to calculating HRV parameters, artifacts and extrasystoles should be removed from the ECG record as they reduce the possibility of spectral analysis of the recording and reduce the likelihood of adequate determination of the state of the vegetative regulation of cardiac activity. For this reason, the RR intervals obtained from the Holter monitoring (Georgieva-Tsaneva, 2012), are determined by the sequence of normal $\mathrm{NN}$ intervals by removing the artifacts and extrasystoles.

In this study the following parameters in the time domain are investigated:

- The average value of RR intervals (ms);

- SDNN (ms) - standard deviation of normal to normal intervals;

- SDANN (ms) - standard deviation of averages normal to normal intervals;

- RMSSD (ms) - root mean square of the successive differences;

- pNN50 (\%) - percent of intervals of successive NN intervals greater than $50 \mathrm{~ms}$.

Data

The investigated data were obtained by means of a Holter monitoring of 24 hour ECG records, an approximate number of RR intervals per one day and one night: 90000 beats.

Long-term 24-hour Holter monitoring studies in 54 healthy subjects (divided into 3 age groups of 18 individuals) were conducted.

Statistical analysis

A Statistical analysis (one-way ANOVA test from summary data) was applied to the obtained parameters. A significance level $\mathrm{p}<0.05$ was considered for comparing the results statistically.

\section{Results}

Table 1 shows the computed statistical data for HRV (MeanRR, SDNN, SDANN, RMSSD, PNN50) and the results of the conducted DFA analysis (alfa1 и alfa2) through the averages of the conducted surveys. The results obtained are shown as Mean \pm standard deviation.

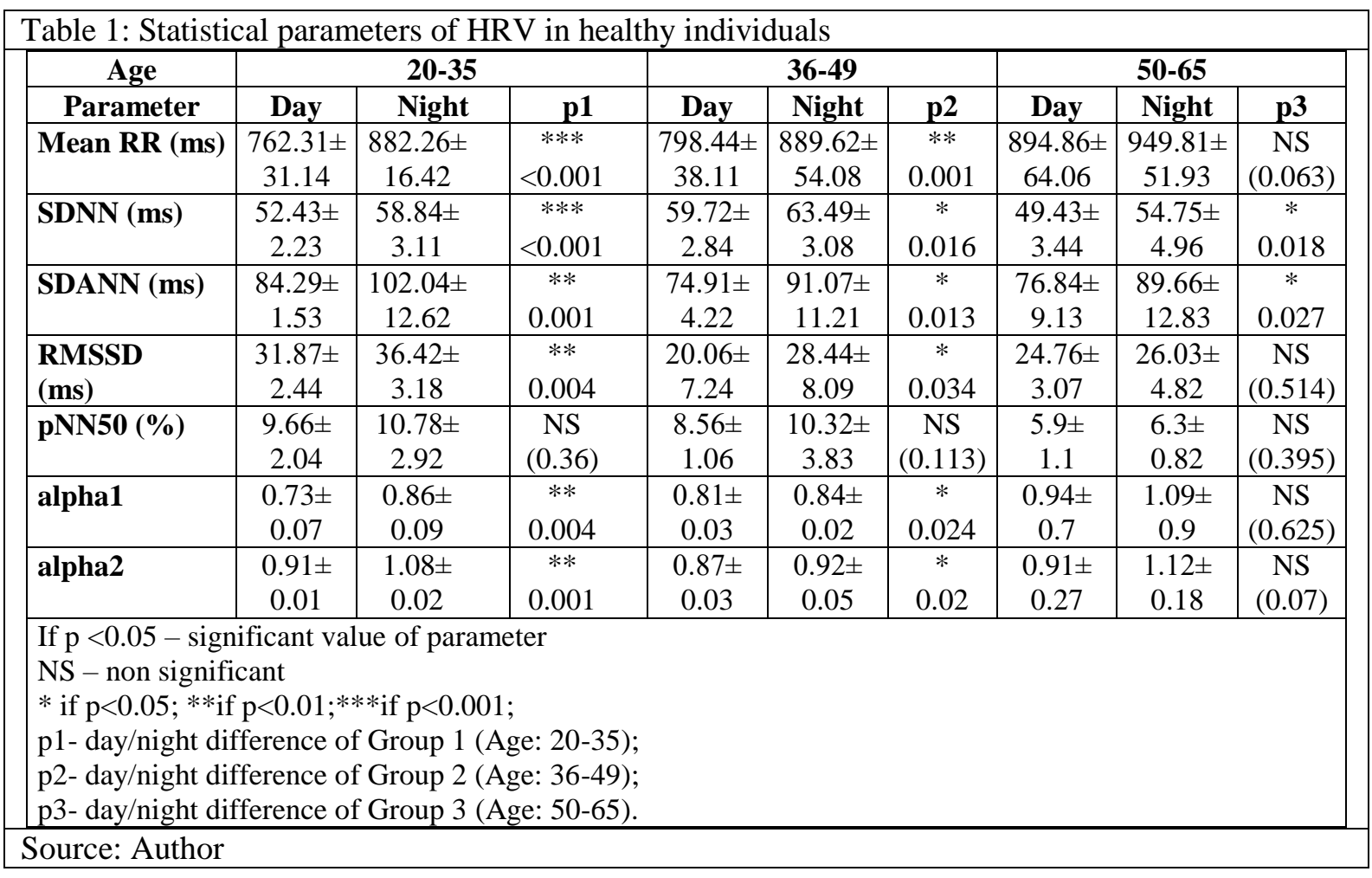

Studies were carried out on the values of the statistical parameters and the parameters obtained with DFA from the day monitoring and night monitoring for the three observed age groups: group 1 (young individuals - 20 to 35 years); group 2 (middle-aged subjects - 36 to 49 years) and group 3 (adult subjects - 50 to 65 years). Using the parameter $\mathrm{p} 1$ (ANOVA test), the ability to distinguish the tested parameters of group 1 during waking (at day) and during sleep (at night) is assessed. The parameter p2 refers to group 2; the parameter $\mathrm{p} 3$ - to group 3. In the group of young people, almost all indicators vary day / night, with significant differences; in the middle group there are differences, but lower $\mathrm{p} 2$ values are 
reported, whereas in the group of the eldest, this difference is greatly reduced (only two SDNN and SDANN indicators differ significantly). The pNN50 parameter does not differ significantly in the three age groups and cannot be an indicator for distinguishing groups.

The highest age group (50-65) shows a decrease in SDNN, SDANN, RMSSD; and during the day and night in absolute value, RR intervals increased (compared to the youngest age group).

The two investigated parameters with DFA are alpha1 and alpha2. The numerical values obtained for these parameters show significant differences between daily and night values in the younger study group (20 to 35 years), differences between daily and night correlations are also reported for the average group (smaller than the younger group), while in the oldest group there were no significant differences between the daily and night values of alpha1 and alpha2, respectively. This shows lower adaptability of the adults to the day-night cycle change.

Figure 1 shows the DFA results for a healthy young individual. The two investigated parameters are alpha1 (shown in cyan color) and alpha2 (shown in red color). The obtained parameters for short correlation values are: alpha $1=0.73$ and for long correlation alpha $2=0.98$. Figure 1 shows higher values for the alpha 2 parameters.

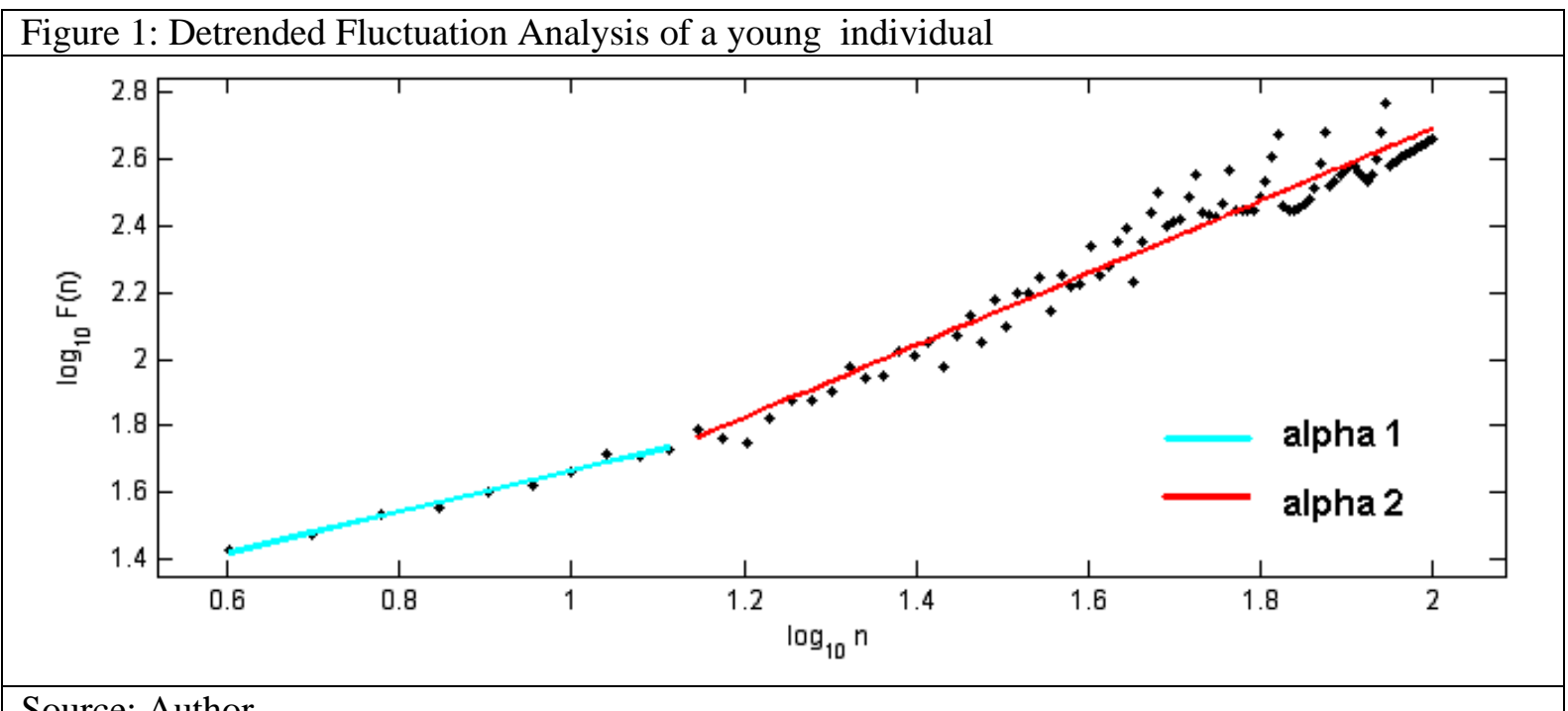

Source: Author

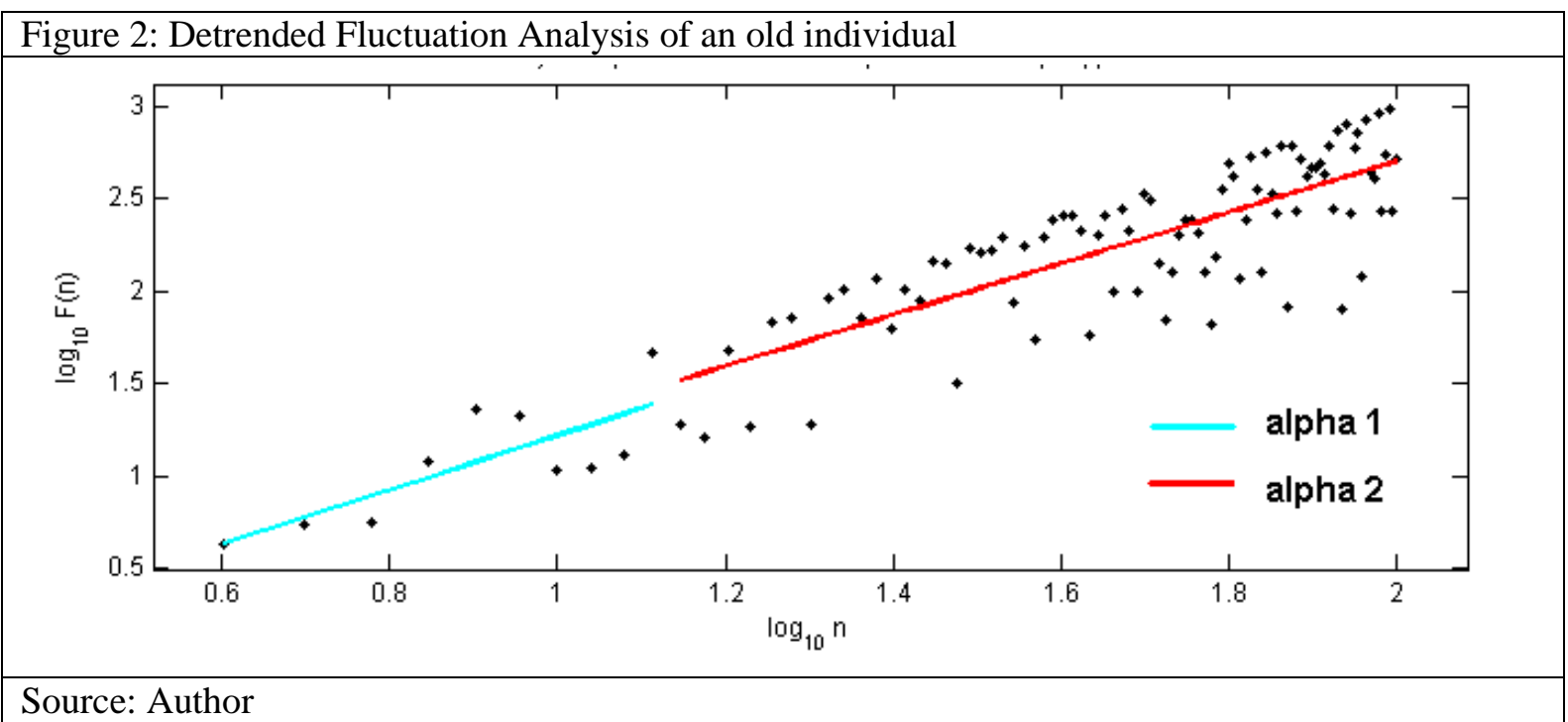

Figure 2 shows the DFA results for a healthy individual of the third age group. The obtained values for the short correlations are as follows: alpha $1=1.09$ and long correlations alpha $2=1.12$. Figure 2 shows that the obtained values of the two parameters alpha1 and alpha2 are almost the same, indicating that as the age increases, the short-term and long-term correlations in the HRV series are aligned. 
The resulting graphical and tabular results show that with the increase in age, the statistical indicators of HRV: Mean RRs are increasing; SDNN, SDANN, RMSSD, pNN50 decreases in the oldest group compared to the youngest group. The alpha1 and alpha2 indicators show significant day and night differences in the young and middle age group, while there are no significant differences in the age group.

\section{Conclusion}

The paper presents the results of studies performed on the Heart Rate Variability of healthy individuals divided into 3 age groups: 20 to 35 years; 36 to 49 years and 50 to 65 years. The purpose of this study is to determine the occurrence of changes in HRV with the change in the age of the people. Surveys are carried out in the time domain through statistical parameters and with a nonlinear method through the application of a Detrended Fluctuation Analysis.

The investigation was performed on 24 hours of ECG records received via Holter monitoring. The results of the youngest group showed significant differences in variability during wakefulness and sleeping. Studies on the oldest group show very small differences in daytime and nighttime parameters and even a lack of them, indicating a decrease in HRV with increasing age and decreasing the ability of the human body to adapt to the day / night transition.

The research done in the report shows that the Detrended Fluctuation Analysis is a suitable method for determining short- and long-term correlations in the cardiology series.

The presented results in the paper can be used by physicians in their practice to assess age-related changes in human individuals.

\section{Acknowledgements}

This research was carried out as part of the project "Investigation of the application of new mathematical methods for the analysis of cardiac data" No. KP-06-N22/5, 07.12.2018, founded by National Science Found, Bulgaria.

\section{References}

Acharya R.U., Lim C. M., Joseph P. (2002). Heart rate variability analysis using correlation dimension and Detrended Fluctuation Analysis, ITBM-RBM, 333-339.

Georgieva-Tsaneva G. (2018). Heart Rate Variability Generating based on Mathematical Tools, Proceedings of the 19th International Conference on Computer Systems and Technologies - CompSysTech'18, (Eds. B. Rachev, A. Smrikarov), September 13-14, Ruse, Bulgaria, 134-138.

Georgieva-Tsaneva G, (2012). QRS detection algorithm for long term Holter records. Proceedings of 14th International Conference on Computer Systems and Technologies-CompSysTech'13 (Eds. B. Rachev, A. Smrikarov), ACM New York, NY, USA, 112-119.

Golińska A. K. (2012). Detrended Fluctuation Analysis in biomedical signal processing: selected examples. Studies in Logic, Grammar, and Rhetoric, 29(42), 107-115. Retrieved from: http:logika.uwb.edu.pl.

Gospodinov M, Gospodinova E, Domuschiev I, Dey N, Ashour A. (2016) Nonlinear analysis of Heart Rate Variability in Type 2 diabetic patients. Fractal Geometry and Nonlinear Analysis in Medicine and Biology, (1), 134-139.

Gronwald T., Hoos O., Hottenroff K. (2019). Effects of a short-term cycling interval session and active recovery on nonlinear dynamics of cardiac autonomic activity in endurance-trained cyclists, Journal of clinical medicine, 8, 194- 204.

Haller A. (1757). Elementa physiologiae corporis humani. In 8 t. - Lausanne: S. d' Arnay, t. 2, lib. 6., 330-332. Retrieved from: http://bowenvanleuveren.tk/download/QmKCgXptjGcC-elementa-physiologiae-corporis-humani-auctore-alberto-vhaller-tomus-primus-octavus

Hon E. H., Lee S. T. (1963). Electronic evaluations of the fetal heart rate patterns preceding fetal death, Further observations. American Journal of Obstetrics and Gynecology, 87, 814-826.

Krishnam R1, Chatlapalli S, Nazeran H, Haltiwanger E, Pamula Y. (2005). Detrended Fluctuation Analysis: A Suitable Long-term Measure of HRV Signals in Children with Sleep Disordered Breathing. Conference proceedings of the IEEE Engineering in Medicine and Biology Society, 2, 1174-7.

Lee J.M., Kim D.J. Kim I.Y. et al. (2002). Detrended Fluctuation Analysis of EEG in sleep apnea using MIT/BIH polysomnography data, Computers in Biology and Medicine, 32, 37-47.

Leite, F. S., Rocha A, Carvalho J. (2010). MATLAB Software for Detrended Fluctuation Analysis of Heart Rate Variability. Biosignals 2010, International Conference on Bio-inspired Systems and Signal Processing, 225-229.

Lin L., Lo M., Ko P., Lin C., Chiang W., Liu Y., Hue K., Lin J., Chen W., Ma M. (2010). Detrended fluctuation analysis predicts successful defibrillation for out-of-hospital ventricular fibrillation cardiac arrest, 81(3), 297-301.

Mahon NG, Hedman AE, Padula M, Gang Y, Savelieva I, Waktare JEP, Malik MM, Huikuri HV, McKenna WJ. (2002).

Fractal correlation properties of R-R interval dynamics in asymptomatic relatives of patients with dilated cardiomyopathy. The European Journal of Heart Failure, 4, 151-158. 
Malik M. 1996. Task Force of the European Society of Cardiology and the North American Society of Pacing and Electrophysiology, Heart rate variability: standards of measurement, physiological interpretation, and clinical use. Circulation, 93, 1043-1065.

Miao T., Sato H., Hashiguchi T., Maruyama I. (2006). Nonlinear analysis of heart rate variability and plethysmogram in subjects with normal and abnormal cardiovascular function. Proceedings of IEEE International Conference on Systems, Manand Cybernetics, 4, 2885-2890.

Nakamura T, Horio H, Miyashita S, Chiba Y, SatoS. (2005). Identification of development and autonomic nerve activity from heart rate variability in preterminfants. Bio Systems, 79, 117-124.

Peng C.K., Havlin S., Stanley H.E., Goldberger A.L. (1995). Quantification of scaling exponents and crossover phenomena in nonstationary heartbeat time series, Chaos, 5, 82-87.

Rojo-Alvarez JL, Sanchez-Sanchez A, Barquero-Perez O, Goya-Esteban R, Everss E, Mora-Jimenez I, Garcia-Alberola A. (2007). Analysis of Physiological Meaning of Detrended Fluctuation Analysis in Heart Rate Variability Using a Lumped Parameter Model. Computers in Cardiology, 34, 25-28.

Šikner T., Janoušek O., Kolář R. (2015). Detrended Fluctuation Analysis of HRV: Comparison of classic and frequencybased parameters. Elektrorevue, 6(2), 30-36.

Valencia JF, Vallverdú M, Schroeder R, Voss A, Cygankiewicz I, Vázquez R, Bayés de Luna A, Caminal P. (2009). Detrended Fluctuation Analysis of Heart Rate by Means of Symbolic Series, Computers in Cardiology, 36, 405-408.

Yeh R., Shieh J., Han Y., Wang. Y., Tseng S. (2006). Detrended Fluctuation Analysis of short-term heart rate variability in surgical intensive care units. Biomedical engineering-applications, basis \& communications, 18, 67-72. 